\section{E-040 ENDOVASCULAR RECONSTRUCTIVE TREATMENT USING A TUNNELING TECHNIQUE FOR A FUSIFORM VERTEBRAL ARTERY DISSECTING ANEURYSM WITH IPSILATERAL DOMINANCE}

${ }^{1} \mathrm{~T}$ Lee*, ${ }^{2} \mathrm{~J}$ Ko, 'S Sung. 'Radiology, Pusan National University Hospital, Busan, Korea, republic of; ${ }^{2}$ Neurosurgery, Pusan National University Hospital, Busan, Korea, republic of

\subsection{6/neurintsurg-2019-SNIS. 115}

Purpose Fusiform dissecting aneurysms involving the dominant vertebral artery with poor collaterals are challenging to treat. This study aimed to evaluate the safety and efficacy of a tunneling technique for reconstructive endovascular treatment of these conditions.

Materials and methods Thirteen patients, 11 men and two women, with a mean age of 54.5 years, each with a fusiform vertebral artery dissecting aneurysm not amenable to internal trapping of the parent artery, underwent reconstructive endovascular treatment using a tunneling technique between Jan 2012 and Dec 2015. The safety, feasibility, and clinical and angiographic outcomes of these procedures were retrospectively evaluated.

Results The average maximum diameter of the fusiform aneurysms was $12.1 \mathrm{~mm}$. Five of them were ruptured. Three aneurysms were treated with a single stent and the remaining ten aneurysms required double stent placement. Treatment was technically successful in all 13 patients, achieving complete occlusion $(\mathrm{n}=10,76.9 \%)$ and near complete occlusion $(n=3,23.1 \%)$. No procedure-related complications occurred in any patient. There were no delayed thromboembolic or hemorrhagic complications during the follow-up period (mean, 19.0 months). Angiographic follow-ups (mean, 9.1 months) showed stable occlusion in 90.9\% (10/11) and asymptomatic in-stent occlusion in one patient $(9.1 \%, 1 / 11)$. At the end of the observation period (mean, 19.0 months), all patients had excellent clinical outcomes (mRS 0, 92.3\%, 12/13), except one ( $\mathrm{mRS} 4$ ), resulting from poor preoperative status.

Conclusion This retrospective study demonstrated that endovascular reconstruction using a tunneling technique was a technically safe, feasible, and clinically effective treatment method for fusiform vertebral artery dissecting aneurysms with ipsilateral dominance.

Disclosures T. Lee: None. J. Ko: None. S. Sung: None.

\section{E-041 ANEURYSM REMODELING FOLLOWING FLOW DIVERSION MITIGATES RISK OF SUBARACHNOID HEMORRHAGE}

G Kaur*, J Cooper, F Al-Mufti, C Gandhi, J Santarelli. Neurosurgery, Westchester Medical Center, Valhalla, NY

\subsection{6/neurintsurg-2019-SNIS.116}

Background Flow diversion has revolutionized treatment of wide-neck aneurysms in the past decade. While the ideal aim of flow diversion is complete elimination of aneurysm filling, with parent vessel reconstruction, some cases result in incomplete occlusion, especially those involving a branch emerging from the aneurysmal sac. The rupture risk of such aneurysms is unknown. We followed patients with pipeline reconstruction but incomplete aneurysmal occlusion, to evaluate long-term subarachnoid hemorrhage risk.
Methodology All patients who were treated at Westchester Medical Center between 2012-18, undergoing elective placement of the Pipeline Flex flow diverter, with at least 1 follow up angiogram 6 months after the device placement, were screened. Patients with incomplete occlusion of the aneurysm in the follow up angiogram were included in this series. All patients were followed until Feb 2019 for subarachnoid hemorrhage. Demographic data and aneurysmal characteristics were derived from chart review. Angiographic remodeling without occlusion was defined as continued aneurysm filling in the neck, reduced or unchanged compared to control angiography, 6+ months after initial treatment. In patients with multiple angiograms, the last angiogram showing persistent aneurysmal filling was used.

Results A total of 225 patients treated with pipeline flex flow diversion from January 2012-December 2018 were screened. 30 aneurysms with incomplete occlusion on follow up angiography were included in this series. Mean age was 60 years (29-76 years), with a male to female ratio of 1:3. The majority (21/30) of the aneurysms with incomplete occlusion were adjacent to an emerging vessel, of which 7 were para-ophthalmic, 6 Pcom, $3 \mathrm{MCA}$, and 5 PICA. The remaining 9 aneurysms were without emerging branches and included 6 supraclinoid ICA and 3 intradural cavernous ICA aneurysms. The mean size of the aneurysm before pipeline embolization was $7.8 \mathrm{~mm}$ (range 2-18 mm). All aneurysms adjacent to an emerging vessel were classified as Raymond class 5. All aneurysms showed a decrease in size following flow diversion, with a mean residual of $2.3 \mathrm{~mm}(1-11 \mathrm{~mm})$. Incomplete occlusion was seen on the angiogram at a mean of 13 months (6-30 months). Zero cases of subarachnoid hemorrhage were noted, during a mean follow up of 33 months (8-87 months).

Conclusion The use of Pipeline flow diversion to treat cerebral aneurysms significantly reduces subsequent risk of subarachnoid hemorrhage, even in cases of incomplete aneurysm occlusion. In our series, the long-term risk of aneurysmal subarachnoid hemorrhage in remodeled but uncured aneurysms was zero percent. This group of aneurysms typically, although not exclusively, involves a locally emerging branch vessel with continued vascular demand. While the goal of Pipeline treatment is to cure the aneurysm, we propose that the process of remodeling following flow diversion appears to confer protection in aneurysms with continued filling.

Disclosures G. Kaur: None. J. Cooper: None. F. Al-Mufti: None. C. Gandhi: None. J. Santarelli: None.

\section{E-042 CEREBRAL HYPERPERFUSION SYNDROME FOLLOWING ENDOVASCULAR CEREBRAL ANEURYSM STENT- COILING: CASE REPORTS AND DIAGNOSTIC CHALLENGES}

${ }^{1} \mathrm{P}$ Yager ${ }^{*},{ }^{2} \mathrm{~L} \mathrm{Lin},{ }^{3} \mathrm{~J}$ Fraser, ${ }^{2} \mathrm{~A}$ Alhajeri. ${ }^{1}$ College of Medicine, University of Kentucky, Lexington, $K Y_{;}{ }^{2}$ Department of Radiology, University of Kentucky, Lexington, $K Y_{i}$ ${ }^{3}$ Department of: Radiology, Neurosurgery, Neurology, Neuroscience, University of Kentucky, Lexington, $K Y$

\subsection{6/neurintsurg-2019-SNIS.117}

Introduction Cerebral hyperperfusion syndrome (CHS) has been reported sparsely after aneurysm clipping and is likely underreported after endovascular treatments. We report two 